\title{
Polaron dynamics in a two-dimensional anharmonic Holstein model
}

\author{
Zolotaryuk, Yaroslav; Christiansen, Peter Leth; Juul Rasmussen, Jens
}

\section{Published in:}

Physical Review B

Link to article, DOI:

10.1103/PhysRevB.58.14305

Publication date:

1998

Document Version

Publisher's PDF, also known as Version of record

Link back to DTU Orbit

Citation (APA):

Zolotaryuk, Y., Christiansen, P. L., \& Juul Rasmussen, J. (1998). Polaron dynamics in a two-dimensional anharmonic Holstein model. Physical Review B, 58(21), 14305-14319.

https://doi.org/10.1103/PhysRevB.58.14305

\section{General rights}

Copyright and moral rights for the publications made accessible in the public portal are retained by the authors and/or other copyright owners and it is a condition of accessing publications that users recognise and abide by the legal requirements associated with these rights.

- Users may download and print one copy of any publication from the public portal for the purpose of private study or research.

- You may not further distribute the material or use it for any profit-making activity or commercial gain

- You may freely distribute the URL identifying the publication in the public portal

If you believe that this document breaches copyright please contact us providing details, and we will remove access to the work immediately and investigate your claim. 


\title{
Polaron dynamics in a two-dimensional anharmonic Holstein model
}

\author{
Y. Zolotaryuk \\ Department of Mathematical Modelling, Technical University of Denmark, DK-2800 Lyngby, Denmark \\ and Department of Mathematics, Heriot-Watt University, Edinburgh EH14 4AS, United Kingdom \\ P. L. Christiansen \\ Department of Mathematical Modelling, Technical University of Denmark, DK-2800 Lyngby, Denmark \\ J. Juul Rasmussen \\ Risb National Laboratory, Optics and Fluid Dynamics Department, P.O. Box 49, 4000 Roskilde, Denmark
}

(Received 24 March 1998)

\begin{abstract}
A generalized two-dimensional semiclassical Holstein model with a realistic on-site potential that contains anharmonicity is studied. More precisely, the lattice subsystem of anharmonic on-site oscillators is supposed to have a restricting core. The core plays the role of an effective saturation nonlinearity for the polaron (selftrapped) solutions. We apply the "logarithmic" potential approximation which allows us to use effectively a variational approach, on one hand, and to study the realistic situation of the potential core and saturation nonlinearity, on the other hand. Analytical estimates suggest the existence of wide polarons, contrary to the case with harmonic on-site potential. Numerical simulations confirm these estimates and show stability of such polaron solutions. We develop a numerical technique which allows us to obtain the profile of extended moving polarons. Simulations show that these polarons can propagate for long distances on the plane retaining their shape and velocity. Collision effects of the two-dimensional polarons are also investigated.
\end{abstract}

[S0163-1829(98)02645-9]

\section{INTRODUCTION}

It is more than 60 years since Landau ${ }^{1}$ presented the idea about self-localization or self-trapping of an excess electron in a polarized field created by itself. Later, on the basis of this idea, Pekar ${ }^{2}$ introduced the notion of a polaron as an extra electron or a hole localized within a potential well that it creates by displacing the atoms (ions) that surround it. The polaron concept is ubiquitous in physics and a lot of studies have been performed during the past half-century, including the contributions by Fröhlich, ${ }^{3}$ Holstein, ${ }^{4}$ Toyozawa, ${ }^{5}$ Rashba, ${ }^{6}$ Emin and Holstein ${ }^{7,8}$ Davydov and Kislukha, ${ }^{9}$ Scott ${ }^{10}$ and many others. ${ }^{12-14}$

In general, the polaron theory applies to any quantum particle or a quasiparticle interacting with relatively massive atoms (groups of atoms or even molecules) surrounding it. For example, replacing the electron or hole with an exciton, one obtains the theory of the formation and motion of selftrapped excitons. ${ }^{9,10}$ Therefore the term "polaron" is used to denote a wide variety of excitations being self-localized through interactions with optical $2^{2,4,13,14}$ and/or acoustic ${ }^{5,9,10}$ phonons of a lattice. However, it should be noticed that ions in real materials are damped and subject to finite temperature (stochastic forcing). As a rule, disorder and different defects are also present in real crystals. Obviously, all these factors destroy any coherent polaron transport. Moreover, when the electron-phonon coupling is sufficiently strong compared to the intersite exchange interaction, the pure polaron states have small size and they appear to be pinned to the lattice. In this case there is no coherent polaron motion as well and only the small-polaron hopping mechanism occurs due to temperature. ${ }^{8}$ Nevertheless, the research on the dynamics of pure self-trapped states, essentially in higher dimensions, continues at an accelerating pace.

An explosion of interest in movable polarons has arisen beginning from the pioneering paper by Davydov and Kislukha ${ }^{9}$ after which the polarons (both acoustic and optical) were often called solitons as mobile objects that maintain dynamical integrity by balancing the effects of nonlinearity (electron-phonon or exciton-phonon coupling) against those of dispersion (exchange or resonance intersite interaction) during their uniform propagation. However, all these studies, except for the recent paper by La Magna et al., ${ }^{15}$ were carried out only in one dimension. On the other hand, the generalizations of the lattice models to higher dimensions ${ }^{16-19}$ are necessary because of natural interest to real two- and three-dimensional crystalline systems. In particular, it is appealing to investigate the polaron dynamics in multidimensional lattices interacting with extra electrons. Emin and Holstein ${ }^{7}$ using scaling arguments in the continuum limit and Kalosakas et ll $^{20}$ applying a discrete variational approach, analyzed the Holstein model with the shortrange (local) electron-lattice interaction ${ }^{4,7}$ within the adiabatic theory. They have shown that in one dimension the standing polaron is always a ground state of the electronlattice system, so that there is a continuous transition from the small-polaron regime obtained for strong electronphonon coupling to the large-polaron regime at weak coupling. In the former case the width of the self-trapped state (the electron wave function and the accompanied lattice deformation) is of few lattice sites only while in the latter one the polaron state extends to lengths significantly larger than the lattice spacing. The similar situation takes place for Davydov's soliton, ${ }^{11,12}$ in general, for the one-dimensional 
acoustic polaron. ${ }^{21}$ In higher dimensions these results drastically change: for sufficiently strong electron-lattice coupling small (quite narrow) polarons exist, but when the coupling decreases, the polaron disappears. ${ }^{20}$ Since the small polaron is pinned to the lattice, it cannot propagate. However, as shown in the present paper, when a saturable anharmonicity is taken into account for the on-site oscillators in the Holstein model, the polaron width can be extended significantly, resulting in the uniform polaron propagation on the twodimensional or three-dimensional lattice. The saturation effect means that the motion of ions is bounded, e.g., while displacing from equilibria, the ions have a finite amplitude including the case of strong electron-phonon coupling. Such a limited ion motion can happen if the potential for ions has some core, so that when an ion approaches the core, its energy tends to infinity. The saturation of the ion (atom) displacements causes the saturation of the nonlinearity of the electron-lattice interaction. Note that the saturable nonlinearity has recently been used to describe successfully the propagation of radially symmetric self-focused light beams. ${ }^{22-28}$

The present paper aims at studying both analytically and numerically the propagation of the polaron on the twodimensional square lattice in the framework of the semiclassical Holstein model. The on-site potential of the model is generalized to have a restricting core that does not allow the lattice ions (atoms) to displace at any distance as in the harmonic version of the model. The core plays the role of the saturable nonlinearity for the formation of self-trapped states. We apply a "logarithmic" approximation for the onsite potential that still has a core being close to the realistic situation, on one hand, and allows us to use effectively a variational approach, on the other hand. Analytical estimates suggest the existence of the polarons with large width, contrary to the case with the harmonic on-site potential. ${ }^{20} \mathrm{Nu}$ merical simulations confirm these estimates and show stability of such polaron solutions. We develop a numerical scheme which allows us to obtain the profile of moving polarons with large extent. The simulations of the equations of motion show that these polarons can propagate for long distances on the lattice retaining their shape and velocity.

The paper is organized as follows. In the next section we derive the equations of motion that describe the coupled system: an excess electron interacting with classical oscillators on a two-dimensional (2D) lattice. In Sec. III we study standing polaron solutions analytically, using variational approximations, and numerically. Moving polaron solutions are obtained and studied numerically in Sec. IV. The polaron collision is also investigated in this section. Finally, some concluding remarks are given in Sec. V.

\section{THE MODEL AND BASIC EQUATIONS}

We consider a square lattice of noninteracting particles of mass $M$, the equilibria of which are situated at the sites labeled by the 2D vector $(m, n)$ with $m$ and $n$ running over all the integers. Each of these particles is subjected to an anharmonic on-site potential $V$ of the realistic shape shown in Fig. 1 (see curve 1), forming the on-site classical nonlinear oscillator. When an external electron (or, in general, a quantum quasiparticle) is added to such a 2D lattice, each of the oscillators is supposed to interact with this electron locally.

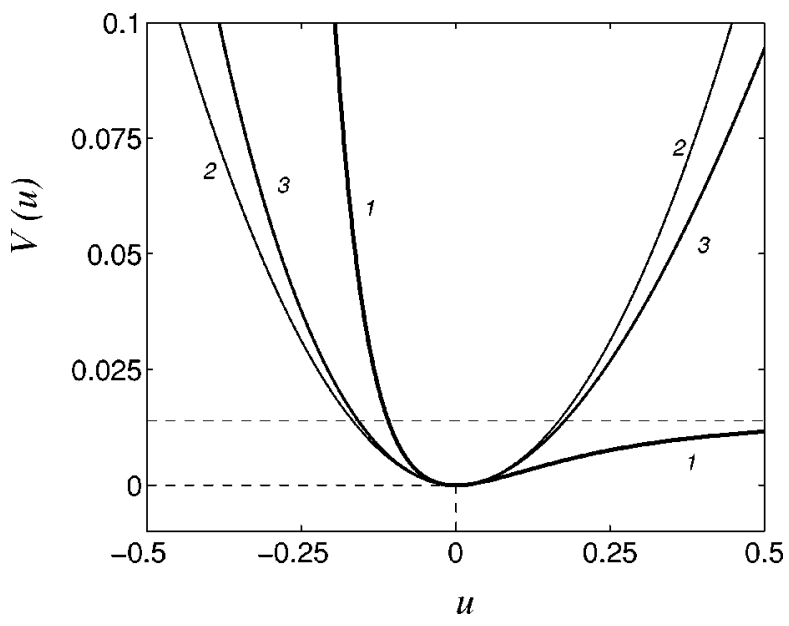

FIG. 1. The shape of the $(12,6)$ LJ potential (curve 1) and of its two approximations: harmonic (curve 2) and logarithmic (curve 3).

This is the issue of the standard semiclassical Holstein model $^{4}$ with local electron-phonon coupling when the anharmonicity of the lattice subsystem is included. In the adiabatic limit the Lagrangian function of such a lattice interacting with an excess electron can be written in the form

$$
\begin{aligned}
\mathcal{L}= & \sum_{m, n}\left\{\psi _ { m n } ^ { * } \left[i \hbar \dot{\psi}_{m n}-\left(E_{0}+\chi Q_{m n}\right) \psi_{m n}\right.\right. \\
& \left.+J\left(\psi_{m-1, n}+\psi_{m, n-1}+\psi_{m+1, n}+\psi_{m, n+1}\right)\right] \\
& \left.+\frac{1}{2} M \dot{Q}_{m n}^{2}-M a^{2} \omega_{0}^{2} V\left(Q_{m n} / a\right)\right\},
\end{aligned}
$$

where the dots denote differentiation with respect to time $t$ and the complex-valued lattice field $\psi_{m n}(t)$ (the coefficient functions of the one-electron state vector) describes the probability amplitude to find the electron at the $(m, n)$ th lattice site, so that it must be normalized to unity:

$$
\sum_{m, n}\left|\psi_{m n}(t)\right|^{2}=1
$$

The constant $J>0$ is the exchange (overlapping) integral that describes the probability of the electron hopping from one lattice site to any of its nearest-neighbor ones, $E_{0}$ is the on-site electron energy when the lattice is undistorted, i.e., when the particles of the lattice are found in their equilibria and $\chi>0$ is the coupling constant of the electron-lattice interaction. The states of the lattice subsystem are described by the real-valued lattice field $Q_{m n}(t)$, each of $Q_{m n}$ being the displacement of the $(m, n)$ th lattice particle from its equilibrium position. The dimensionless on-site potential $V(u)$ is normalized by the relations $V(0)=0$ and $V^{\prime \prime}(0)=1$, so that the constant $\omega_{0}=\sqrt{K / M}$, with $K$ being the elastic constant, is the characteristic frequency of each on-site oscillator (the eigenfrequency of small-amplitude oscillations of lattice particles). We consider the realistic form of the potential $V(u)$ with a core which prevents the nearest-neighbor lattice particles from approaching each other on very close distances. It could be the standard $(12,6)$ Lennard-Jones (LJ) potential 


$$
V(u)=\frac{1}{72}\left[(1+u)^{-6}-1\right]^{2}, \quad-1<u<\infty,
$$

shown in Fig. 1 by curve 1 . However, any potential that has two cores, one for $u<0$ and the other for $u>0$, seems to be even more realistic. Since $\chi>0$, the total energy of the system goes down [see the Lagrangian function (1)], if all or some of $Q_{m n}$ become negative. Therefore only the left branch of the potential $V(u)$ is of interest when self-trapped states are considered. The distance between the core and the equilibrium position is normalized to unity, so that the particle displacements from the equilibria are measured in the units of the constant $a$ being the maximally possible negative displacement of the lattice particles (the characteristic length). This constant should be significantly less than the lattice spacing $l$.

The form of the potential (3) is very inconvenient for analytical studies and therefore its harmonic approximation shown in Fig. 1 by curve 2 is commonly used in the polaron theory. ${ }^{4,7,20}$ Here we introduce another approximation which keeps the main feature of any realistic potential, namely, the presence of a core, and allows us to perform some analytical studies, at least, for the static polaron solutions. This approximation is chosen in the following form:

$$
V(u)=u-\ln (1+u)=\sum_{j=2}^{\infty} \frac{(-u)^{j}}{j}
$$

with the expansion series being valid in the interval $-1<u$ $\leqslant 1$. We call the potential (4) the "logarithmic" approximation illustrated in Fig. 1 by curve 3 . The first term of the series (4) is the harmonic approximation. From the comparison of the shapes of the three potentials depicted in Fig. 1 one can conclude that the logarithmic approximation is much better than the harmonic one, particularly, in the region of negative values of the variable $u$. Note that the polaron (selftrapped) states are formed with only negative displacements $Q_{m n}$, so that the polaron theory deals only with this region of the lattice displacements.

There are two convenient ways to rewrite the Lagrangian (1) or the corresponding equations of motion in dimensionless form. One of these is to use the characteristic time scale in the electron subsystem, namely, to introduce the scaled time as $\tau=J t / \hbar$. In this paper we use the characteristic time scale for the lattice subsystem and therefore define the scaled (dimensionless) time by

$$
\tau=\omega_{0} t
$$

Then it is convenient to introduce the new (dimensionless) lattice fields

$$
\phi_{m n}(\tau)=\psi_{m n}(t) \exp \left[\frac{i}{\hbar}\left(E_{0}-4 J\right) t\right], \quad u_{m n}(\tau)=\frac{Q_{m n}(t)}{a}
$$

and to rewrite the Lagrangian function (1) in the following (dimensionless) form:

$$
\begin{aligned}
L=\frac{\mathcal{L}}{J} & =\sum_{m, n}\left\{\phi _ { m n } ^ { * } \left[(i / \sigma) \frac{d \phi_{m n}}{d \tau}+\phi_{m+1, n}+\phi_{m, n+1}\right.\right. \\
& \left.-4 \phi_{m n}+\phi_{m-1, n}+\phi_{m, n-1}-\alpha u_{m n} \phi_{m n}\right] \\
& \left.+\frac{\alpha}{\beta}\left[\frac{1}{2}\left(\frac{d u_{m n}}{d \tau}\right)^{2}-V\left(u_{m n}\right)\right]\right\}
\end{aligned}
$$

with the three characteristic dimensionless parameters

$$
\alpha=\frac{\chi a}{J}, \quad \beta=\frac{\chi}{M a \omega_{0}^{2}}=\frac{\chi}{K a}, \quad \sigma=\frac{J}{\hbar \omega_{0}} .
$$

Then the corresponding Euler-Lagrange equations are written as follows:

$$
\begin{gathered}
(i / \sigma) \frac{d \phi_{m n}}{d \tau}=-\left(\phi_{m+1, n}+\phi_{m, n+1}-4 \phi_{m n}\right. \\
\left.+\phi_{m-1, n}+\phi_{m, n-1}\right)+\alpha u_{m n} \phi_{m n}, \\
\frac{d^{2} u_{m n}}{d \tau^{2}}=-V^{\prime}\left(u_{m n}\right)-\beta\left|\phi_{m n}\right|^{2},
\end{gathered}
$$

and the normalization condition (2) becomes

$$
\sum_{m, n}\left|\phi_{m n}(\tau)\right|^{2}=1
$$

Each of the parameters $\alpha, \beta$, and $\sigma$ defined by Eqs. (8) has the definite physical meaning. Thus, according to the linear Schrödinger equation (9), in which the displacement field $u_{m n}$ forms a potential well caused by the lattice deformation, the parameter $\alpha$ measures the depth of this well (given by the electron-phonon coupling constant $\chi$ ) compared to its width (given by the electron dispersion constant $J$ ). Therefore the parameter $\alpha$ describes the magnitude of the electron trapping by lattice deformation. In contrast, the parameter $\beta$ measures the response of the electron on the lattice. According to the linear lattice equation (10) with the source created by the electron, the parameter $\beta$ describes the magnitude of the source that distorts the lattice, i.e., "digs" a potential well for itself. The third parameter $\sigma$ is a dynamical one; it disappears in the static theory and measures the ratio of characteristic time scales of both the subsystems (electron and lattice).

Let us now evaluate the possible values of the parameters $\alpha, \beta$, and $\sigma$ which are reasonable from the physical point of view. Using some data described by $\operatorname{Scott}^{10}$ as well as other data from the references therein, we may choose the following characteristic values: $a=0.1 \AA, J=5 \mathrm{~cm}^{-1}, M=m_{p}$ where $m_{p}$ is the proton mass, $\omega_{0}=10^{13} \mathrm{~s}^{-1}$, and $\chi$ $=(2-6) \times 10^{-11}$ Newtons. If we take, for instance, $\chi=2$ $\times 10^{-11}$ Newtons, then the dimensionless constants $\alpha, \beta$, and $\sigma$ calculated according Eqs. (8) take the values: $\alpha$ $=2.0, \beta=12.0$, and $\sigma=0.94$. These values or a little bit bigger will be used below in our numerical calculations. Note that the increase of the exchange interaction $J$ to the values more reasonable for crystals leads to increasing the polaron size and therefore to higher polaron movability. 
We need to have also the general expression for the Hamiltonian function (the total energy) of our system. To write it, we define the following conjugate momenta:

$$
\Pi_{m n}=\frac{\partial L}{\partial\left(d \phi_{m n} / d \tau\right)}=\frac{i}{\sigma} \phi_{m n}^{*},
$$

$$
P_{m n}=\frac{\partial L}{\partial\left(d u_{m n} / d \tau\right)}=\frac{d u_{m n}}{d \tau},
$$

where the Lagrangian function $L$ is given by Eq. (7). Then the dimensionless Hamiltonian function (in units of $J$ ) takes the form

$$
\begin{aligned}
H & =H\left\{\Pi_{m n}, \phi_{m n} ; P_{m n}, u_{m n}\right\}=\sum_{m, n}\left(\Pi_{m n} \frac{d \phi_{m n}}{d \tau}+P_{m n} \frac{d u_{m n}}{d \tau}\right)-L \\
& =\sum_{m, n}\left\{\phi_{m n}^{*}\left[-\left(\phi_{m+1, n}+\phi_{m, n+1}-4 \phi_{m n}+\phi_{m-1, n}+\phi_{m, n-1}\right)+\alpha u_{m n} \phi_{m n}\right]+\frac{\alpha}{\beta}\left[\frac{1}{2}\left(\frac{d u_{m n}}{d \tau}\right)^{2}+V\left(u_{m n}\right)\right]\right\} .
\end{aligned}
$$

We look for the solutions of the equations of motion (9) and (10) in the form of a modulated plane wave propagating in any direction on the 2D lattice given by the wave vector $\mathbf{k}=\left(k_{1}, k_{2}\right)$. Therefore we substitute the ansatz

$$
\phi_{m n}(\tau)=\varphi_{m n}(\tau) \exp \left\{i\left[m k_{1}+n k_{2}-\sigma\left(\varepsilon_{0}+\varepsilon\right) \tau\right]\right\},
$$

where

$$
\varepsilon_{0}=2\left(2-\cos k_{1}-\cos k_{2}\right)
$$

is the free-electron energy band, into the basic equations presented above. Thus, the equations of motion (9) and (10) are reduced to the three equations:

$$
\begin{aligned}
& -\cos k_{1}\left(\varphi_{m+1, n}-2 \varphi_{m n}+\varphi_{m-1, n}\right) \\
& -\cos k_{2}\left(\varphi_{m, n+1}-2 \varphi_{m n}+\varphi_{m, n-1}\right)+\alpha u_{m n} \varphi_{m n}=\varepsilon \varphi_{m n},
\end{aligned}
$$$$
\frac{d \varphi_{m n}}{d \tau}=-\sigma\left[\sin k_{1}\left(\varphi_{m+1, n}-\varphi_{m-1, n}\right)\right.
$$$$
\left.+\sin k_{2}\left(\varphi_{m, n+1}-\varphi_{m, n-1}\right)\right],
$$$$
\frac{d^{2} u_{m n}}{d \tau^{2}}=-V^{\prime}\left(u_{m n}\right)-\beta \varphi_{m n}^{2} .
$$

Using next the normalization condition [see Eqs. (11) and (14)]

$$
\sum_{m, n} \varphi_{m n}^{2}=1
$$

the Lagrangian and Hamiltonian functions (7) and (13) are transformed to

$$
\begin{aligned}
L= & -\sum_{m, n}\left\{\cos k_{1}\left(\varphi_{m+1, n}-\varphi_{m n}\right)^{2}\right. \\
& +\cos k_{2}\left(\varphi_{m, n+1}-\varphi_{m n}\right)^{2}+\alpha u_{m n} \varphi_{m n}^{2}-\varepsilon \varphi_{m n}^{2} \\
& \left.-\frac{\alpha}{\beta}\left[\frac{1}{2}\left(\frac{d u_{m n}}{d \tau}\right)^{2}-V\left(u_{m n}\right)\right]\right\},
\end{aligned}
$$

$$
\begin{aligned}
H= & \sum_{m, n}\left\{\cos k_{1}\left(\varphi_{m+1, n}-\varphi_{m n}\right)^{2}\right. \\
& +\cos k_{2}\left(\varphi_{m, n+1}-\varphi_{m n}\right)^{2}+\alpha u_{m n} \varphi_{m n}^{2} \\
& \left.+\frac{\alpha}{\beta}\left[\frac{1}{2}\left(\frac{d u_{m n}}{d \tau}\right)^{2}+V\left(u_{m n}\right)\right]\right\},
\end{aligned}
$$

respectively.

The dimensionless energy $\varepsilon<0$ of binding the electron to a lattice deformation is the spectral parameter of the stationary 2D Schrödinger equation (16). This parameter is to be found together with the lattice deformation field. However, it can be expressed in terms of both the lattice fields $\varphi_{m n}$ and $u_{m n}$ as follows. Indeed, multiplying both sides of Eq. (16) by $\varphi_{m n}$, summing them over all $(m, n)$ 's and using the normalization condition (19), we obtain

$$
\begin{aligned}
\varepsilon= & \sum_{m, n}\left[\cos k_{1}\left(\varphi_{m+1, n}-\varphi_{m n}\right)^{2}\right. \\
& \left.+\cos k_{2}\left(\varphi_{m, n+1}-\varphi_{m n}\right)^{2}+\alpha u_{m n} \varphi_{m n}^{2}\right] .
\end{aligned}
$$

On the other hand, the total energy $E$ of the electron-phonon system [i.e., the Hamiltonian function (21)] can be expressed in terms of the binding energy $\varepsilon$ as

$$
E=H=\varepsilon_{0}+\varepsilon+\frac{\alpha}{\beta} \sum_{m, n}\left[\frac{1}{2}\left(\frac{d u_{m n}}{d \tau}\right)^{2}+V\left(u_{m n}\right)\right],
$$

where $\varepsilon_{0}$ is given by Eq. (15). Therefore the total energy $E$ is split into the two parts: the electron and lattice ones, each of these consisting of both the kinetic and potential energies.

To conclude this section, we consider the limiting case when the lattice is undistorted $\left(u_{m n} \equiv 0\right)$. In this limit the binding energy $\varepsilon \rightarrow 0$ and, according to the linear dispersion law (15), the group velocity in the electron subsystem is

$$
\mathbf{v}=\frac{J l}{\hbar} \frac{\partial \varepsilon_{0}}{\partial \mathbf{k}}=\frac{2 J l}{\hbar}\left(\sin k_{1}, \sin k_{2}\right)
$$

with $l$ being the lattice spacing. Therefore we may define the dimensionless velocity

$$
\mathbf{s}=\mathbf{v} / l \omega_{0}=2 \sigma\left(\sin k_{1}, \sin k_{2}\right)
$$


which describes the propagation velocity of the linear waves of electron probability if the lattice is undistorted.

\section{STANDING 2D POLARON SOLUTIONS}

First we consider the particular case of standing (static) solutions for the logarithmic potential (4). Using that $d^{2} u_{n} / d \tau^{2}=0$, from Eq. (18) [see also Eq. (10)] we obtain

$$
u_{m n}=-\frac{\beta\left|\phi_{m n}\right|^{2}}{1+\beta\left|\phi_{m n}\right|^{2}}=-\frac{\beta \varphi_{m n}^{2}}{1+\beta \varphi_{m n}^{2}} .
$$

Inserting this expression into the Lagrangian (20) or the Hamiltonian (21) and using that $k_{1}=k_{2}=0$ for the static case [see Eq. (17)], we find that the energy of the system can be written in the form

$$
\begin{aligned}
E= & -L=\sum_{m, n}\left[\left(\varphi_{m+1, n}-\varphi_{m n}\right)^{2}\right. \\
& \left.+\left(\varphi_{m, n+1}-\varphi_{m n}\right)^{2}-(\alpha / \beta) V\left(\beta \varphi_{m n}^{2}\right)\right]
\end{aligned}
$$

where the potential $V$ is given by Eq. (4) and the term with the spectral parameter (binding energy) $\varepsilon$ has been omitted because it is constant due to the normalization condition (19). Next, substituting the solution (26) into Eq. (16), we get the discrete nonlinear Schrödinger (DNLS) equation of the form

$$
\begin{gathered}
\varphi_{m+1, n}+\varphi_{m, n+1}-4 \varphi_{m n}+\varphi_{m-1, n}+\varphi_{m, n-1} \\
+g \varphi_{m n}^{3} /\left(1+\beta \varphi_{m n}^{2}\right)+\varepsilon \varphi_{m n}=0,
\end{gathered}
$$

where the parameters $\alpha$ and $\beta$ in the nonlinear term appear in the form of the product which we denote by

$$
g=\alpha \beta=\chi^{2} / J M \omega_{0}^{2} .
$$

When the constant $\beta \rightarrow 0$, Eq. (28) is reduced to the standard DNLS equation with cubic nonlinearity which corresponds to the harmonic Holstein model. In this limit both the parameters are "sticked" together, forming only one characteristic parameter $g$, which can be referred to as the selftrapping coupling constant. Indeed, it describes both the effects in the self-trapping mechanism: (i) the capture of an electron by the potential well of the lattice deformation field $(\alpha)$ and (ii) the creation of the potential well by the electron acting as an external force $(\beta)$. However, in the anharmonic case, this mutual proportionality is broken because the influence of the electron on the lattice becomes nonlinear as seen from the equation of motion (18).

In the opposite limit $\beta \rightarrow \infty$, the nonlinear term in the DNLS equation (28) is transformed to the linear one, so that the nonlinearity in this equation disappears and, as a result, the localization effect should diminish. In other words, the nonlinearity is saturated and one should expect that wide (extended) polaron solutions can exist as well, contrary to the harmonic Holstein model which admits either very narrow polaron solutions or completely extended (delocalized) states. ${ }^{20}$ Therefore, the core anharmonicity leads to the DNLS equation with the saturation nonlinearity. It follows from Fig. 1 that realistic potentials should reveal the saturation effect even more.
To study analytically the polaron solutions to Eqs. (16) and (18) with the constraint (19), we use a variational approach, using both a discrete trial function defined on the 2D lattice and a 2D continuous trial function. Each of these functions is chosen to have only one variational parameter describing the size of localization. Substituting a discrete trial function into the expression for the energy (27) or a continuous trial function into the continuum version of this energy, we shall obtain a corresponding function with respect to the variational parameter which can be minimized and its optimal value can be calculated.

\section{A. Discrete variational approximation}

In this subsection we use the discrete variational approach, assuming the exponentially decreasing behavior of a trial 2D lattice function for the field $\varphi_{m n}$ normalized by the condition (19). From the symmetric point of view the following two "opposite" positions of the polaron center should be considered: (i) the polaron is located exactly at a lattice site and (ii) at the middle point between the four nearestneighbor lattice sites (the central point of the lattice cell). Intuitively, the on-site position (i) seems to have lower energy. However, this should be checked. For this first case we consider the discrete normalized trial function ${ }^{20}$

$$
\varphi_{m n}=A q^{|m|+|n|}, \quad A=A(q)=\frac{1-q^{2}}{1+q^{2}},
$$

with the variational parameter $q, 0<q<1$, which determines how strongly the polaron is localized. The substitution of this trial function into the expression (27) yields

$$
\begin{aligned}
E= & 4 \frac{(1-q)^{2}}{1+q^{2}} \\
& -\alpha\left\{1-\beta^{-1}\left[\ln \left(1+\beta A^{2}\right)+4 \sum_{n=1}^{\infty} \ln \left(1+\beta A^{2} q^{2 n}\right)\right.\right. \\
& \left.\left.+4 \sum_{m, n=1}^{\infty} \ln \left(1+\beta A^{2} q^{2 m+2 n}\right)\right]\right\}
\end{aligned}
$$

For the polaron states centered in the middle of the lattice cell we consider the second ansatz as follows:

$$
\varphi_{m n}=\frac{1}{2} \vartheta(q)\left(1-q^{2}\right) q^{|m|+|n|},
$$

where $\vartheta(q)=q^{-2}$ if $m \geqslant 1$ and $n \geqslant 1, \vartheta(q)=q^{-1}$ if $m \leqslant 0$ and $n \geqslant 1$ or $m \geqslant 1$ and $n \leqslant 0$, and $\vartheta(q)=1$ if $m \leqslant 0$ and $n$ $\leqslant 0$. Substituting this ansatz into Eq. (27), we obtain the second expression for the variational energy $E$ :

$$
\begin{aligned}
E= & 2(1-q)^{2}-\alpha\left[1-4 \beta^{-1}\right. \\
& \left.\times \sum_{m, n=1}^{\infty} \ln \left(1+\frac{\beta}{4}\left(1-q^{2}\right)^{2} q^{2 m+2 n-4}\right)\right] .
\end{aligned}
$$




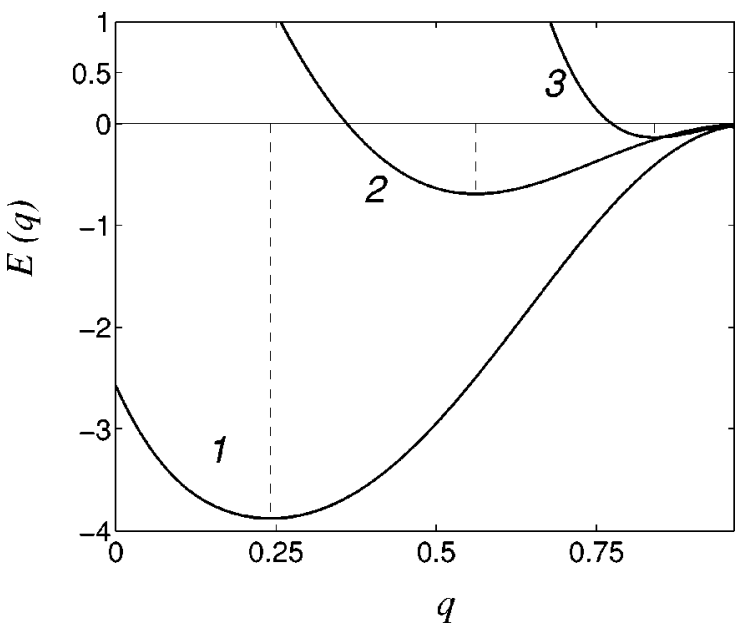

FIG. 2. The total energy $E(q)$ given by Eq. (31) and plotted as a function of the variational parameter $q$ for the following three sets of parameter values: $\alpha=7$ and $\beta=6$ (curve 1), $\alpha=3$ and $\beta=10$ (curve 2), and $\alpha=1, \beta=20$ (curve 3).

We find the optimal value $q=q_{0}$ from the condition that the energy $E$ attains a minimum at this value. As a result, we have found that the energy (31) is lower than the energy (33). Therefore, in what follows we shall use only the trial function (30). In the harmonic limit the energy (31) is reduced to the expression

$$
E=\frac{4(1-q)^{2}}{1+q^{2}}-\frac{g}{2}\left(1-q^{2}\right)^{2} \frac{\left(1+q^{4}\right)^{2}}{\left(1+q^{2}\right)^{6}}
$$

obtained by Kalosakas et al. ${ }^{20}$ Note that in the limit of extended states $q \rightarrow 1$ and for this case the trial ansatz (30) is transformed to the explicit form which should be written for any finite square domain consisting of $N^{2}$ lattice sites. The normalized function $\varphi_{m n}$ for the uniformly extended states is $\varphi_{m n}=N^{-1}$, so that the energy (31) for this square becomes

$$
E_{N}=\alpha\left[\left(N^{2} / \beta\right) \ln \left(1+\beta / N^{2}\right)-1\right] .
$$

In the limit of the infinite square domain we have $\lim _{N \rightarrow \infty} E_{N}=0$. Therefore, for localized states there should be a certain value $q=q_{0}$ at which the variational energy (31) takes its minimal (negative) value.

The variational energy of the system $E$ given by Eq. (31) is plotted in Fig. 2 as a function of the parameter $q$ for different values of the characteristic parameters $\alpha$ and $\beta$. Let $q=q_{0}=q_{0}(\alpha, \beta)$ be the minimum of each curve $E=E(q)$. Contrary to the results for the harmonic approximation, ${ }^{20}$ the polaron width, which depends drastically on the parameters $\alpha$ and $\beta$, can be quite large. Indeed, we have $q_{0}=0.242$ for $\alpha=7$ and $\beta=12$ (curve 1) which corresponds to the narrow solution, but $q_{0}=0.562$ for $\alpha=3$ and $\beta=10$ (curve 2) which is the intermediate case, and even $q_{0}=0.841$ at $\alpha=1$ and $\beta=20$ (curve 3). The latter set of the parameter values for $\alpha$ and $\beta$ provides a fairly extended profile. The cross section of the $2 \mathrm{D}$ profile $\left|\phi_{m n}\right|=\varphi_{m n}$ at $m=0$ calculated according to the ansatz (30) for these three solutions $q=q_{0}$ is plotted in Fig. 3. Therefore, we have shown, at least, within the logarithmic approximation, the existence of the $2 \mathrm{D}$ polaron solutions admitting, in dependence of the parameters $\alpha$ and $\beta$,

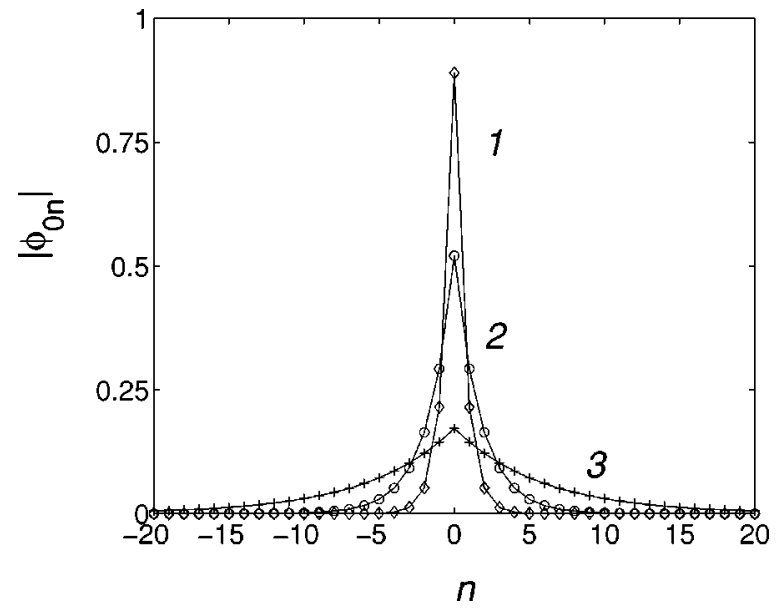

FIG. 3. The $m=0$ section of the polaron profile for the normalized lattice function $\left|\phi_{0 n}\right|=\varphi_{0 n}$ at $\alpha=7, \beta=6$ (curve 1), $\alpha=3$, $\beta=10$ (curve 2), and $\alpha=1, \beta=20$ (curve 3).

the whole spectrum of polaron widths, contrary to the case of the harmonic approximation ${ }^{20}$ where only narrow localized states can exist.

Now let us analyze the role of the parameters $\alpha$ and $\beta$ in the polaron formation more precisely. We have calculated the two dependences of the solution $q=q_{0}$ on $\alpha$ for $\beta$ fixed and, vice versa, $\beta$ for $\alpha$ fixed. The solution $q_{0}=q_{0}(\alpha)$ as a function of $\alpha$ is presented in Fig. 4 for three values of the parameter $\beta$ : $\beta=3$ (curve 1), $\beta=5$ (curve 2), and $\beta=10$ (curve 3). The important result is that the polaron state does not exist for all values of $\alpha$, but only for $\alpha$ greater than a critical value $\alpha_{c}=\alpha_{c}(\beta)$. At $\alpha=\alpha_{c}$ the optimal variational parameter $q_{0}$ attains its maximum value that corresponds to the broad polaron solution and it decreases gradually with the increase of $\alpha$. This means that the polaron is getting more and more narrow when the nonlinear term in Eq. (28) increases, but the saturation parameter $\beta$ is constant. Figure 4 also demonstrates how the critical value $\alpha_{c}$ depends on the parameter $\beta$ : it decreases when $\beta$ increases. This behavior can be explained by the form of the nonlinear term in Eq. (28): the decrease of $\alpha$ and the increase at the same time of

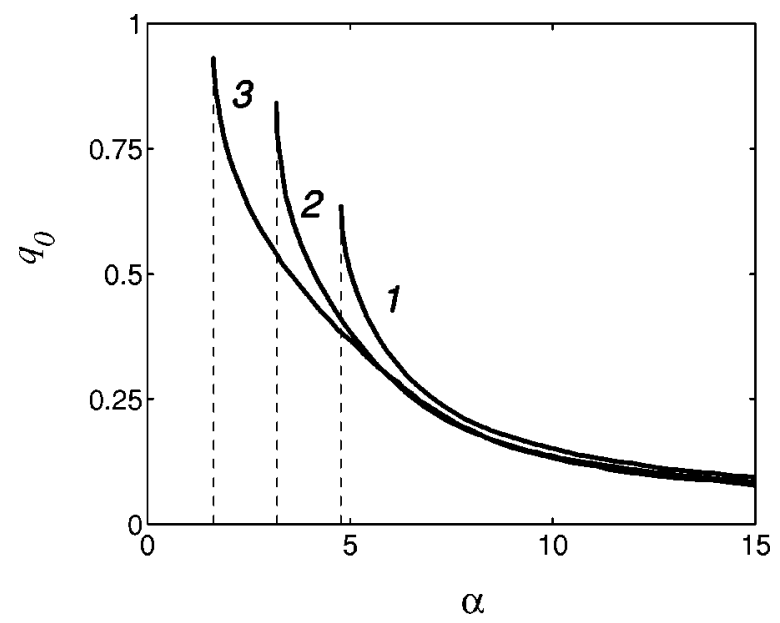

FIG. 4. Optimal value, $q_{0}$, of the variational parameter $q$ as a function of $\alpha$ at the three values of $\beta$ : $\beta=10$ (curve 1), $\beta=5$ (curve 2), and $\beta=3$ (curve 3 ). 


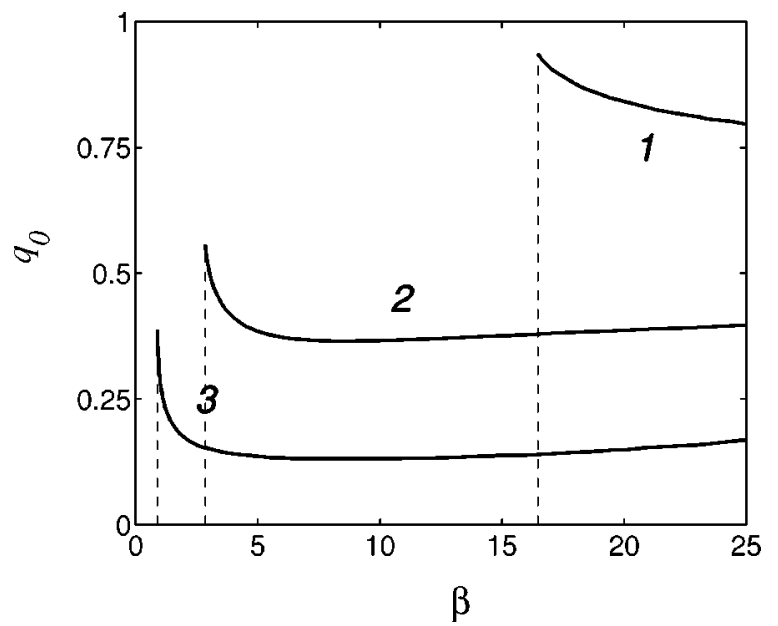

FIG. 5. Optimal value, $q_{0}$, of the parameter $q$ as a function of $\beta$ at the three values of $\alpha: \alpha=10$ (curve 1), $\alpha=5$ (curve 2), and $\alpha$ $=1$ (curve 3$)$.

$\beta$, keeping their product (i.e., the self-trapping parameter $g$ ), enlarges the saturation effect of the nonlinearity, resulting in broadening the existence area of polaron solutions. On the other hand, if $\beta$ is large enough, we do not observe significant changes in the dependence of the solution $q_{0}$ on the parameter $\alpha$.

Similarly to the results illustrated by Fig. 4, for each value of the parameter $\alpha$ a certain critical value $\beta_{c}=\beta_{c}(\alpha)$ can be found, starting from which $\left(\beta>\beta_{c}\right)$ the solution $q_{0}$ $=q_{0}(\beta)$ exists (see Fig. 5). Again, with the decrease of $\alpha$, the critical value $\beta_{c}$ decreases. The polaron becomes narrower because the nonlinear term in the DNLS equation (28) is getting larger if the self-trapping parameter $g$ keeps the same value, but the saturation parameter $\beta$ decreases. For large values of $\beta$ the nonlinearity reaches saturation and therefore the shape of the polaron solution does not change when $\beta$ tends to infinity. Having found both the dependences $\alpha_{c}=\alpha_{c}(\beta)$ and $\beta_{c}=\beta_{c}(\alpha)$, we can plot a diagram curve which separates, on the plane $(\alpha, \beta)$, the areas of existence and nonexistence of the polaron solutions. Such a curve is plotted in Fig. 6. Nearby this curve the polaron profiles being in the existence region are very extended and while crossing this curve, they continuously run to completely delocalized states. Those polaron states which are far away from the existence diagram are quite narrow.

\section{B. A variational approach in the continuum limit}

The discrete approximation described in the previous subsection is supposed to work well for narrow solutions while for broad polarons this approach seems to be crude. In order to check this, it is reasonable to treat our system in the continuum limit, using an appropriate 2D continuous trial function. To compare the results for the $2 \mathrm{D}$ case with those for other dimensions and since the calculations can be easily performed for any dimension $d$, we consider here the general case. Thus, in the continuum limit we substitute the $d$ dimensional lattice vector $\left(n_{1}, \ldots, n_{d}\right)$ by the continuous vector $\left(x_{1}, \ldots, x_{d}\right)$, setting $x_{1}=n_{1}, \ldots, x_{d}=n_{d}$. Then the discrete expression (27) is transformed to

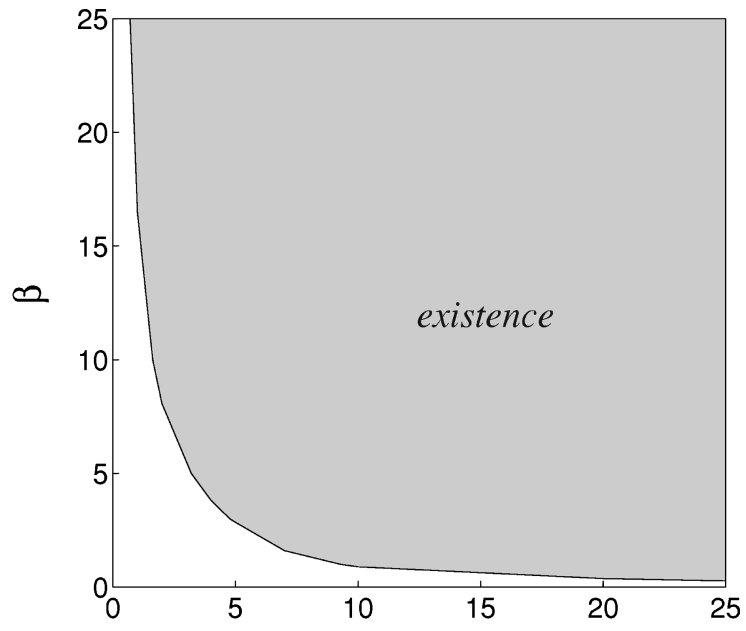

$\alpha$

FIG. 6. Existence diagram; the curve splits the $(\alpha, \beta)$ plane into the two regions: the existence (dark area) and nonexistence (below the curve) of polaron solutions.

$$
\begin{gathered}
E=\int\left[(\nabla \varphi)^{2}-(\alpha / \beta) V\left(\beta \varphi^{2}\right)\right] d x_{1} \ldots d x_{d}, \\
\nabla=\left(\partial_{x_{1}}, \ldots, \partial_{x_{d}}\right) .
\end{gathered}
$$

From the point of view of analytical calculations it is convenient to use the following continuous normalized trial function:

$$
\varphi=\varphi\left(x_{1}, \ldots, x_{d}\right)=(\mu / 2)^{d / 2} \prod_{i=1}^{d} \operatorname{sech}\left(\mu x_{i}\right)
$$

with the variational parameter $\mu$. Using the series expansion (4) and the trial function (37), by straightforward calculations we obtain

$$
E(\mu)=\frac{d}{3} \mu^{2}+\alpha \sum_{j=2}^{\infty} \frac{c_{j}^{d}}{j}\left[-\beta(\mu / 2)^{d}\right]^{j-1},
$$

where the constants $c_{j}$ 's are defined by

$$
c_{j}=\frac{1}{2} \int \operatorname{sech}^{2 j} \zeta d \zeta=\frac{(2 j-2) ! !}{(2 j-1) ! !} .
$$

Since all the coefficients $c_{j}$ are bounded from above, say, beginning from $j \geqslant 4$, we have $c_{j} \leqslant 48 / 105<1 / 2$, the series in the energy (38) is well-defined in the interval $0<\mu$ $\leqslant 2\left(2 \beta^{-1}\right)^{1 / d}$. Therefore for sufficiently small solutions $\mu$ (when the continuum limit indeed can be applied) we can get some reasonable results. Particularly, we can consider the harmonic limit which is easily obtained from the expansion (38) if only the term with $j=2$ is kept. Consequently, taking into account that $c_{2}=2 / 3$, one can write the following expression for the variational energy in the harmonic limit:

$$
E(\mu)=(d / 3) \mu^{2}-(g / 2)(\mu / 3)^{d} .
$$

Similarly, rewriting Eq. (22) in the continuum limit, one can calculate the binding energy $\varepsilon$ in the harmonic approximation: 


$$
\varepsilon(\mu)=(d / 3) \mu^{2}-g(\mu / 3)^{d} .
$$

The total variational energy $E(\mu)$ given by Eq. (40) has a nontrivial minimum only in the $1 \mathrm{D}$ case. This minimum is attained at $\mu_{0}=g / 4$, so that the variational solution for the 1D Holstein model:

$$
\varphi(x)=(g / 8)^{1 / 2} \operatorname{sech}(g x / 4), \quad \varepsilon=-g^{2} / 16
$$

coincides with the exact solution (in the harmonic limit) of the corresponding continuum version of the DNLS equation [see Eq. (28)]

$$
\varphi^{\prime \prime}+g \varphi^{3}+\varepsilon \varphi=0,
$$

where the function $\varphi(x)$ satisfies the continuum version of the normalization condition (19). It is important to note that this solution is valid for sufficiently small values of the coupling parameter $g(g \ll 1)$ when the continuum approximation is applied.

As for the 2D case, the minimum of the energy (40) is indefinite, but it occurs only at the fixed value of the coupling constant: $g=1 / 12$; it takes the zero value, the same as for the delocalized state. There are no minima in higher dimensions. However, if any anharmonicity is involved, a minimum may appear in the two dimensions. Indeed, let us consider the next term in the series expansion (38) when $d$ $=2$. Then we obtain the expression

$$
E(\mu)=\frac{2}{3} \mu^{2}\left(1-\frac{g}{12}+\frac{2}{225} g \beta \mu^{2}\right) .
$$

For the continuum limit to be applied we need the variational parameter $\mu$ to be as small as possible $(\mu \ll 1)$. One can see from expression (44) that this happens if the coupling parameter $g$ exceeds 12, so that the sum of the first two terms is negative and it is close to 1 while the second (positive) term should increase as much as possible. This can be achieved for large values of $\beta$. The minimum of the energy (44) occurs at

$$
\mu_{0}=\frac{15}{2 \beta} \sqrt{\frac{\beta}{12}-\frac{1}{\alpha}} .
$$

Thus, contrary to the $1 \mathrm{D}$ case (where the inequality $g \ll 1$ is required for the existence of wide polaron profiles), the broad 2D polaron solutions can exist only if the inequality $g>12$ is approximately satisfied. Below this will be confirmed numerically by exact results. Therefore, it should be emphasized that the range of the system parameters for which welldefined polaron solutions exist critically depends on spatial dimensionality.

Similarly, one can also calculate the binding energy $\varepsilon$ adding the next term in the series expansion to the harmonic approximation (41). Using then the solution (45), we obtain the dependence

$$
\varepsilon=\varepsilon(\alpha, \beta)=-\frac{25}{4 \beta}\left(\frac{11}{144} g+\frac{5}{g}-\frac{4}{3}\right) .
$$

This energy is negative for all $g>12$ and it decreases linearly (approximately) with the increase of the parameter $\alpha$ at fixed values of $\beta$. It also decreases with the growth of the parameter $\beta$ at fixed values of $\alpha$. This behavior described by the

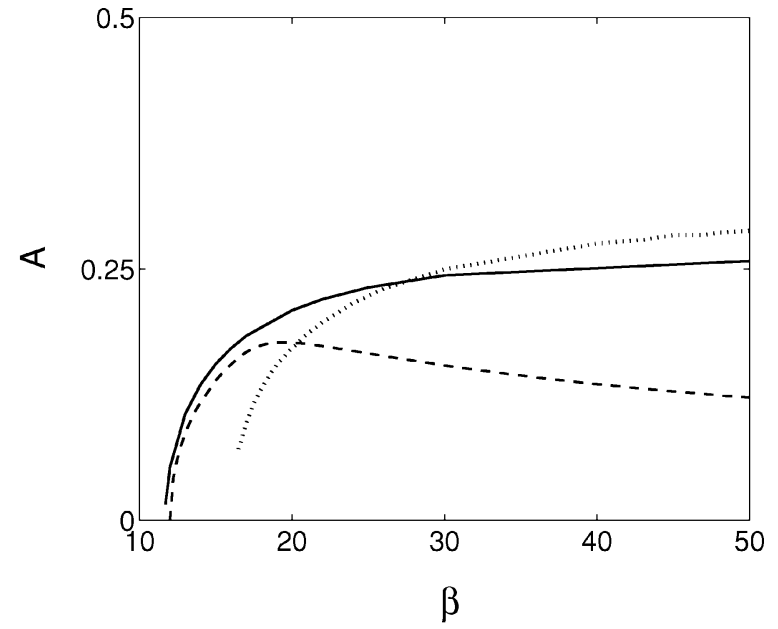

FIG. 7. Amplitude $A$ of the $\varphi_{m n}$ lattice field against the saturation parameter $\beta$ obtained at the value $\alpha=1$ within the discrete variational approach (dotted curve), in the continuum limit (dashed curve), and by minimization (solid curve).

two inequalities $\partial \alpha / \partial \varepsilon<0$ and $\partial \beta / \partial \varepsilon<0$ is in correspondence with the stability criterion proved analytically by Laedke et al. ${ }^{29}$ and confirmed numerically by Christiansen et $a l^{30}$ for the 2D DNLS equation.

\section{Results obtained by minimization}

In the previous two subsections we have studied the system of Eqs. (16) and (18) under the various approximations in order to have an idea about its general features. Now we want to establish how good our variational approximations are and also whether the polaron solutions are stable or not. Exact standing polaron solutions to the problem can be found numerically by minimization of the energy (27) under the constraint (19). This constraint means that the polaron solutions (more precisely, the $\varphi_{m n}$ profile) have to be found on the multidimensional sphere (19). This conditional minimization problem was solved by using the conjugate-gradients method. The results of the discrete variational approximation were used as initial conditions for the minimization procedure. For narrow polaron solutions they appeared to be quite good approximations. The comparison of the exact results obtained by minimization with both the variational approximations is given in Fig. 7. Here we have plotted the amplitude $A=\max \left|\phi_{m n}\right|=\max \varphi_{m n}$ found by these three different techniques: the dotted curve was obtained by the discrete variational procedure and calculated according to Eq. (31), the dashed curve was found using the continuous variational approach and calculated by summing the series (38) with $d$ $=2$, and the solid curve is the numerical solution obtained by minimization. Each of these solutions demonstrates the nonexistence of localized solutions for sufficiently small $\beta$ $<\beta_{c}$. As illustrated by the dashed and solid curves, near the critical value $\beta_{c}$, where the polaron solutions are extended, the results obtained by minimization and the continuous variational method practically coincide. On the other hand, far away from the point $\beta_{c}$, when the polaron solutions become narrow, the discrete variational solution is close to that obtained by minimization (compare the dotted and solid curves). 

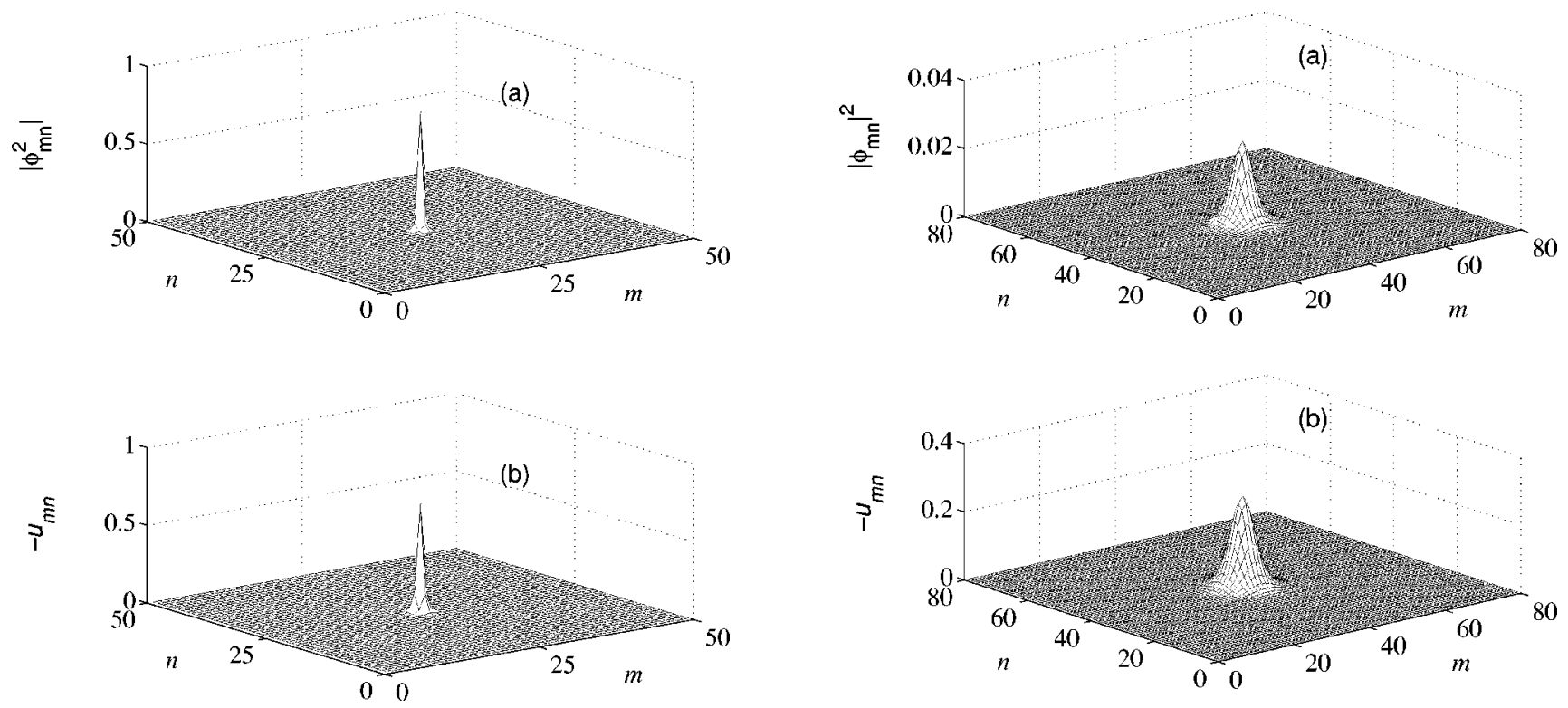

FIG. 8. Narrow profile of the (a) $\left|\phi_{m n}\right|^{2}=\varphi_{m n}^{2}$ and (b) $-u_{m n}$ polaron components obtained by minimization for the parameter values $\alpha=7$ and $\beta=3$.

The $2 \mathrm{D}$ profiles of the $\left|\phi_{m n}\right|=\varphi_{m n}$ and $u_{m n}$ components which were obtained by minimization are shown in Fig. 8. In Fig. 9 we show a direct comparison between the numerically obtained solution and the one obtained by the discrete varia-
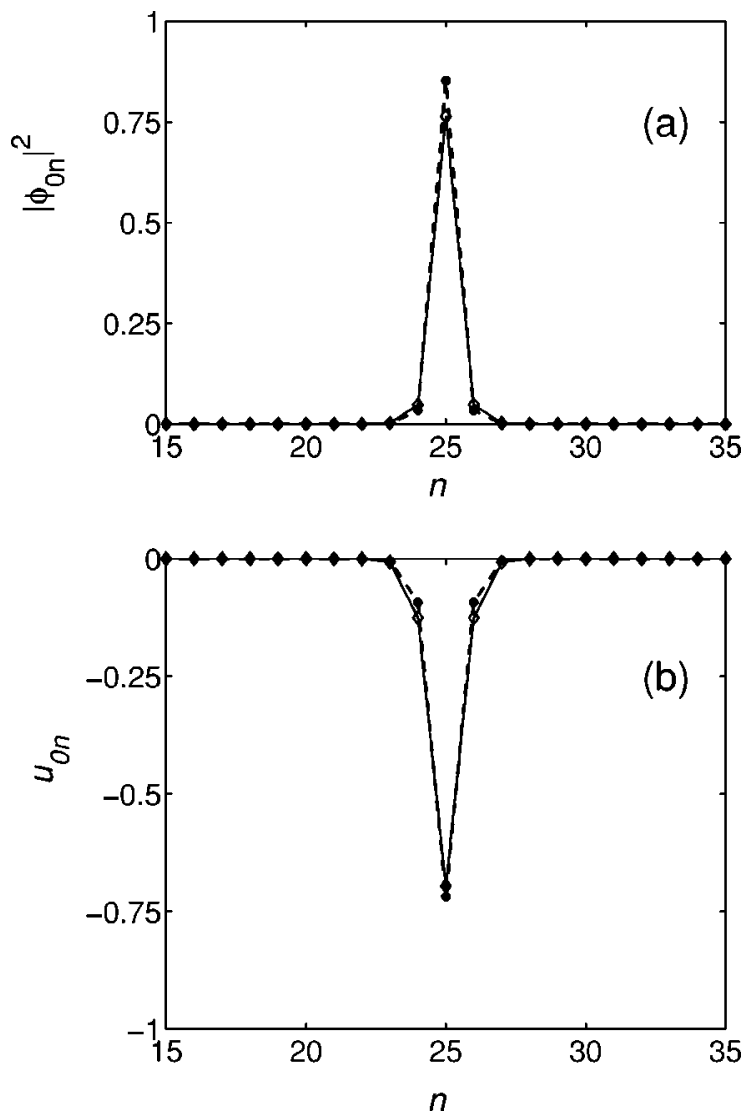

FIG. 9. The $m=0$ section of the polaron profiles obtained within the discrete variational approach (dashed lines) and by minimization (solid lines): the (a) $\left|\phi_{0 n}\right|^{2}=\varphi_{0 n}^{2}$ and (b) $u_{0 n}$ lattice functions; $\alpha=7$ and $\beta=3$.

FIG. 10. Broad profile of the (a) $\left|\phi_{m n}\right|^{2}=\varphi_{m n}^{2}$ and (b) $-u_{m n}$ polaron components obtained by minimization for $\alpha=1$ and $\beta$ $=15$.

tional method for the case in Fig. 8. The agreement of the results is excellent for both the polaron components. Similarly, we examined the continuous variational method. The two-component polaron profile shown in Fig. 10 is quite wide and therefore it is reasonable to compare it with the corresponding results obtained within the continuous variational approach. Such a comparison is presented in Fig. 11 for the $m=0$ section of both the polaron components. Again, the agreement is quite satisfactory. Finally, the polaron profiles obtained by minimization were used as initial data for the simulations of the basic equations of motion (9) and (10), using the fourth-order Runge-Kutta method. The solutions were found to be real stationary; the initial profile did not change during the time evolution of 1000 periods $T$ $=2 \pi / \sigma \epsilon$ of carrier oscillations.

Using the minimization procedure, we have also calculated the dependence of the binding energy $\varepsilon$ on the system parameters $\alpha$ and $\beta$, using the expression [see Eqs. (22) and (26)]

$$
\begin{aligned}
\varepsilon= & \sum_{m, n}\left[\left(\varphi_{m+1, n}-\varphi_{m n}\right)^{2}+\left(\varphi_{m, n+1}-\varphi_{m n}\right)^{2}\right. \\
& \left.-g \varphi_{m n}^{4} /\left(1+\beta \varphi_{m n}^{2}\right)\right] .
\end{aligned}
$$

Particularly, the solid curve in Fig. 12 describes the behavior of $\varepsilon$ as a function of the saturation parameter $\beta$ at a fixed value of $\alpha$. The dashed line in this figure illustrates the approximate dependence (46) which approaches the exact curve for small values of $\beta$, as expected due to taking into account only the cubic term in the series expansion. As mentioned above, the dependence $\varepsilon=\varepsilon(\alpha, \beta)$ is in correspondence with the stability criterion obtained previously ${ }^{29,30}$ in the case of the 2D DNLS equation. Also, the full numerical solutions with the polaron as an initial condition indicate stability. 

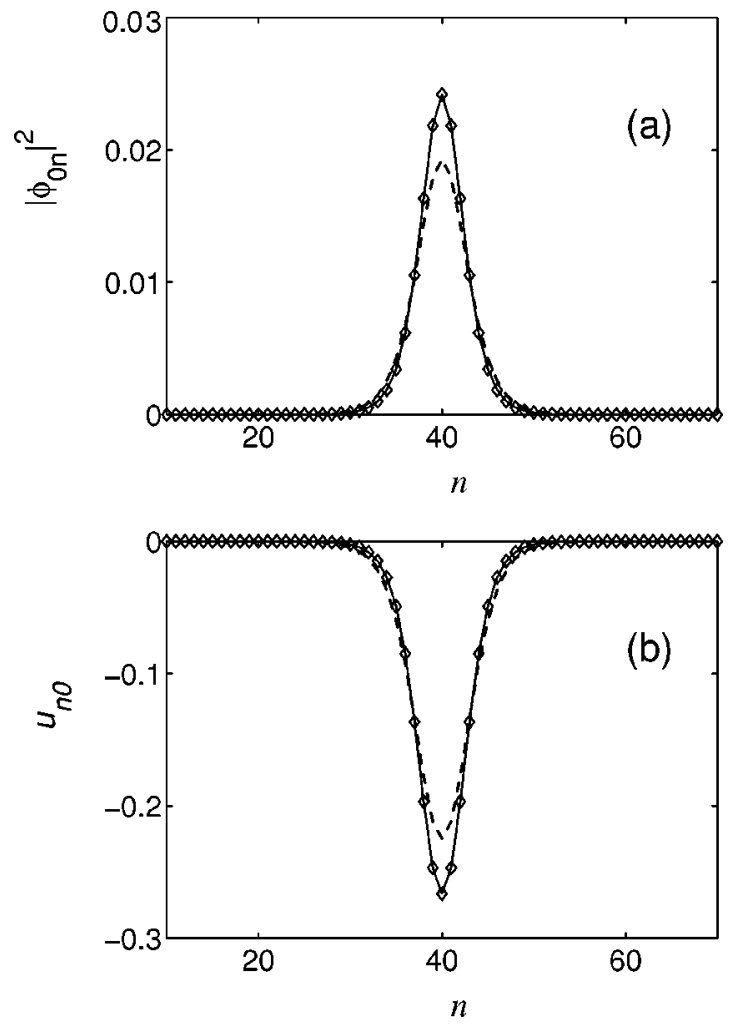

FIG. 11. The $m=0$ section of the polaron profiles obtained within the continuum variational approach (dashed lines) and by minimization (solid lines): the (a) $\left|\phi_{0 n}\right|^{2}=\varphi_{0 n}^{2}$ and (b) $u_{0 n}$ lattice fields; $\alpha=1, \beta=15$, and $\mu_{0}=0.278$.

\section{MOVING 2D POLARONS}

In this section we are interested in the motion of the 2D polarons. Since the polarons with narrow profile are pinned to the lattice, we expect to get their motion when the polaron width is sufficiently large, i.e., in the continuum limit. In fact, we need to have some numerical procedure which would allow us to find for each velocity stationary polaron profiles. Next, whether or not these profiles are stationary, could be checked by direct simulations of the basic equations of motion (9) and (10).

In order to find soliton solutions of large extent in the 1D case which are smooth lattice fields, the numerical procedure is quite simple. ${ }^{31}$ However, it becomes much more sophisticated in higher dimensions. Below we develop this approach by using appropriate discretizations of spatial partial derivatives.

Let us consider the propagation of some stationary profile with a constant velocity $\mathbf{s}=\left(s_{1}, s_{2}\right)$ in the direction given by

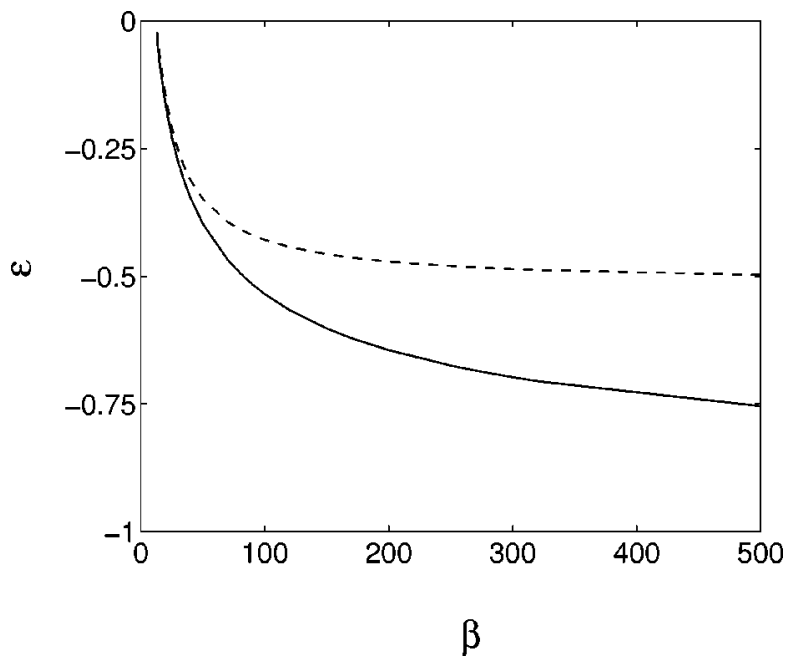

FIG. 12. Binding electron energy $\varepsilon$ as a function of the saturation parameter $\beta$ calculated by minimization (solid line) and in the continuum limit when only the quadratic and cubic terms in the series expansion (4) are kept, $\alpha=1$.

the wave vector $\mathbf{k}=\left(k_{1}, k_{2}\right)$. We set $m=x$ and $n=y$ and for traveling-wave solutions one can write

$\varphi_{m n}(\tau)=\varphi\left(x-s_{1} \tau, y-s_{2} \tau\right), \quad u_{m n}(\tau)=u\left(x-s_{1} \tau, y-s_{2} \tau\right)$

Therefore, using the definition of the dimensionless time $\tau$ given by Eq. (5), we find that the velocity $\mathbf{s}$ is measured in units of $l \omega_{0}$ where $l$ is the lattice spacing, so that $\mathbf{s}=\mathbf{v} / v_{0}$ with $v_{0}=l \omega_{0}$ [the same as for the linear waves, see Eq. (25)]. Moreover, Eq. (17) implies the same one-to-one correspondence between the vectors $\mathbf{s}$ and $\mathbf{k}$ as given by Eq. (25). Indeed, in the continuum limit one can write the following discretization:

$$
\begin{aligned}
\frac{d \varphi_{m n}}{d \tau}= & -\left(s_{1} \partial_{x}+s_{2} \partial_{y}\right) \varphi \\
\simeq & -\frac{1}{2}\left[s_{1}\left(\varphi_{m+1, n}-\varphi_{m-1, n}\right)\right. \\
& \left.+s_{2}\left(\varphi_{m, n+1}-\varphi_{m, n-1}\right)\right] .
\end{aligned}
$$

Comparing Eqs. (17) and (49) gives the same relation (25) valid also for the linear waves of the probability amplitude for the free electron.

For seeking localized solutions of a sufficiently extended profile we use the following representation of the time derivative $d^{2} u_{m n} / d \tau^{2}$ in Eq. (18) by symmetrized 2D secondorder spatial difference derivatives:

$$
\begin{aligned}
\frac{d^{2} u_{n}}{d \tau^{2}}= & \left(s_{1}^{2} \partial_{x}^{2}+2 s_{1} s_{2} \partial_{x} \partial_{y}+s_{2}^{2} \partial_{y}^{2}\right) \varphi \\
\simeq & s_{1}^{2}\left(\varphi_{m+1, n}-2 \varphi_{m n}+\varphi_{m-1, n}\right)+s_{1} s_{2}\left(u_{m+1, n}+u_{m, n+1}-2 u_{m n}-u_{m+1, n-1}-u_{m-1, n+1}+u_{m, n-1}\right. \\
& \left.+u_{m-1, n}\right)+s_{2}^{2}\left(\varphi_{m, n+1}-2 \varphi_{m n}+\varphi_{m, n-1}\right) .
\end{aligned}
$$

Then the difference equations (16) and (18), with the left-hand side replaced by the right-hand side of Eq. (50), are obtained as extremum conditions $\partial \bar{L} / \partial \varphi_{m n}=0$ and $\partial \bar{L} / \partial u_{m n}=0$ of the discretized Lagrangian function 

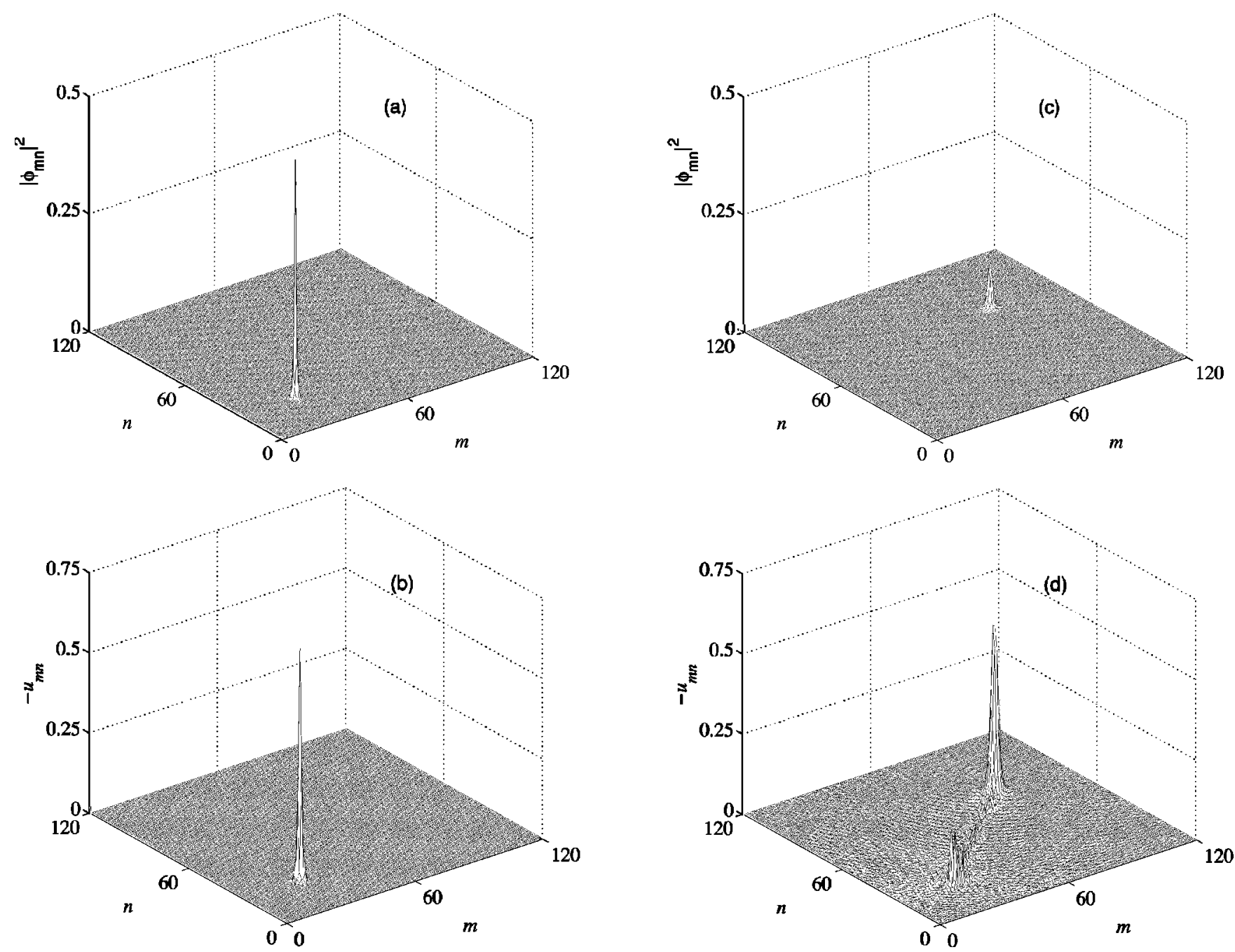

FIG. 13. Nonuniform motion of the narrow polaron accompanied by emission of small-amplitude waves due to its pinning to the lattice for the parameter values $\alpha=4, \beta=5$, and $\sigma=0.2$ : initial (a) $\left|\phi_{m n}(0)\right|^{2}=\varphi_{m n}^{2}(0)$, (b) $-u_{m n}(0)$ and final (c) $\left|\phi_{m n}(300)\right|^{2}=\varphi_{m n}^{2}(300)$, (d) $-u_{m n}(300)$ polaron profiles. The direction of polaron propagation and its velocity are given by the vectors $\mathbf{k}=(0.7,0.5)$ and $\mathbf{s}$ $=(0.258,0.192)$.

$$
\begin{aligned}
\bar{L}= & \bar{L}\left\{\varphi_{m n} ; u_{m n}\right\}=-\sum_{m, n}\left\{\cos k_{1}\left(\varphi_{m+1, n}-\varphi_{m n}\right)^{2}+\cos k_{2}\left(\varphi_{m, n+1}-\varphi_{m n}\right)^{2}+\alpha u_{m n} \varphi_{m n}^{2}\right. \\
& \left.-\frac{1}{2}\left[s_{1}\left(u_{m+1, n}-u_{m n}\right)+s_{2}\left(u_{m, n+1}-u_{m n}\right)\right]^{2}+V\left(u_{m n}\right)\right\} .
\end{aligned}
$$

Note that this procedure is applied only for sufficiently wide polaron profiles.

Similarly to the previous section, we found the profile of moving polaron solutions by minimization. This minimization procedure was performed using the conjugate-gradients method.

\section{A. Single-polaron motion}

The numerical results that describe the motion of a single 2D polaron are presented in the set of Figs. 13 and 14. Thus, the first panels (a,b) of Figs. 13 and 14 represent the initial (at $\tau=0$ ) two-component polaron profiles found by the mini- mization of the function $-\bar{L}$. By an appropriate choice of the system parameters, these profiles were chosen to be sufficiently narrow, in order to demonstrate braking the polaron due to its pinning to the lattice, and extended, to get uniform polaron motion. The final (at $\tau=300$ ) polaron profiles are presented in the second panels $(\mathrm{c}, \mathrm{d})$ of these figures. Figure 13 shows that at the beginning of the motion the polaron loses some part of its velocity, it became wider, and afterwards its motion was stabilized with less velocity and without emission of small-amplitude waves. This part of the polaron kinetic energy was transformed to some breatherlike lattice oscillations that were left at the initial position of the polaron. This indicates that if one forces the narrow state to 

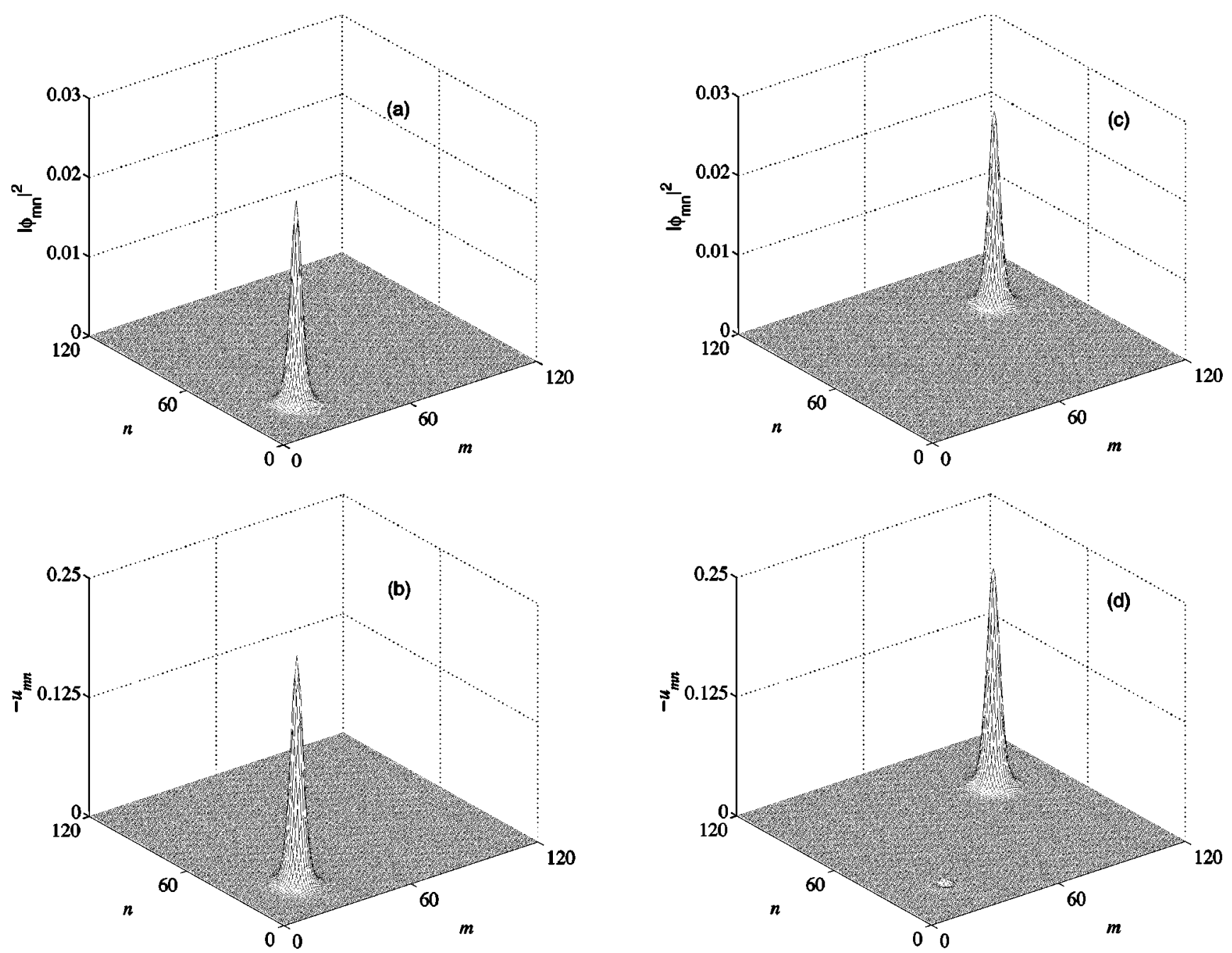

FIG. 14. Uniform motion of the extended polaron in the lattice with $\alpha=1, \beta=12$, and $\sigma=0.2$ : initial (a) $\left|\phi_{m n}(0)\right|^{2}=\varphi_{m n}^{2}(0)$, (b) $-u_{m n}(0)$ and final (c) $\left|\phi_{m n}(300)\right|^{2}=\varphi_{m n}^{2}(300)$, (d) $-u_{m n}(300)$ polaron profiles. The direction of polaron propagation and its velocity are given by the vectors $\mathbf{k}=(0.6,0.5)$ and $\mathbf{s}=(0.226,0.192)$.

move, it tends to transform into a broader stable state which then moves almost uniformly without changing its form. On the other hand, as illustrated in Fig. 14, the wide polaron propagates freely with the constant velocity, retaining its shape and the direction of propagation. The effective mass of a polaron (i.e., the $2 \mathrm{D}$ soliton) $M_{0}$ moving uniformly on the 2D lattice along some direction given by the wave vector $\mathbf{k}$ $=\left(k_{1}, k_{2}\right)$ can be calculated numerically according to the formula

$$
M_{0}=\frac{2 J}{l^{2} \omega_{0 \mathbf{s} \rightarrow 0}^{2}} \lim _{\frac{\mathrm{s}}{\mathbf{s}^{2}}} \frac{E(\mathbf{s})-E(0)}{l^{2} \omega_{0}^{2}} \bar{M}_{0},
$$

where the energy $E(\mathbf{s})$ is calculated according to Eq. (23) and $\bar{M}_{0}$ is the dimensionless polaron mass. Here the effective polaron mass $M_{0}$ has been defined from the expansion of the total energy of a moving polaron into the series with respect to the velocity $\mathbf{v}$ and finding the coefficient at $\mathbf{v}^{2} / 2$, similarly to the procedure of calculating the effective mass of an electron $m_{0}$ in the band (15): $m_{0}=\hbar^{2} / 2 l^{2} J$ with $l$ being the lattice spacing constant. In the units of $J / l^{2} \omega_{0}^{2}$ the (dimensionless) effective electron mass is $\bar{m}_{0}=1 / 2 \sigma^{2}$. For the parameter values $\alpha=1, \beta=14$, and $\sigma=0.2$ we have found $\bar{M}_{0}$ $=12.36$, while the effective electron mass in the band is $\bar{m}_{0}=12.50$. Therefore, we have obtained that the polaron mass is less than the band electron mass. This occurs because the energy level (22) goes down with increasing the polaron velocity $\mathbf{s}$. The value $\bar{M}_{0}=12.36$ has been obtained for the two directions of polaron propagation: along the $m$ axis and the lattice diagonal.

\section{B. Two-polaron interactions}

Now we are interested in the interaction of two polarons on the square lattice. We assume the two electrons to be noninteracting particles, so that we can work in the framework of the model described in Sec. II. However, the physical meaning of the wave function $\psi_{m n}$ becomes a bit different, namely, now we assume $\left|\psi_{m n}\right|^{2}$ to be the probability of two electrons to be found on the site $(m, n)$. Therefore the normalization condition (2) should hold in our simulations. Despite the electrons are assumed to be noninteracting particles, an effective attractive interaction between them appears due to the lattice deformation forming a stable bipo- 

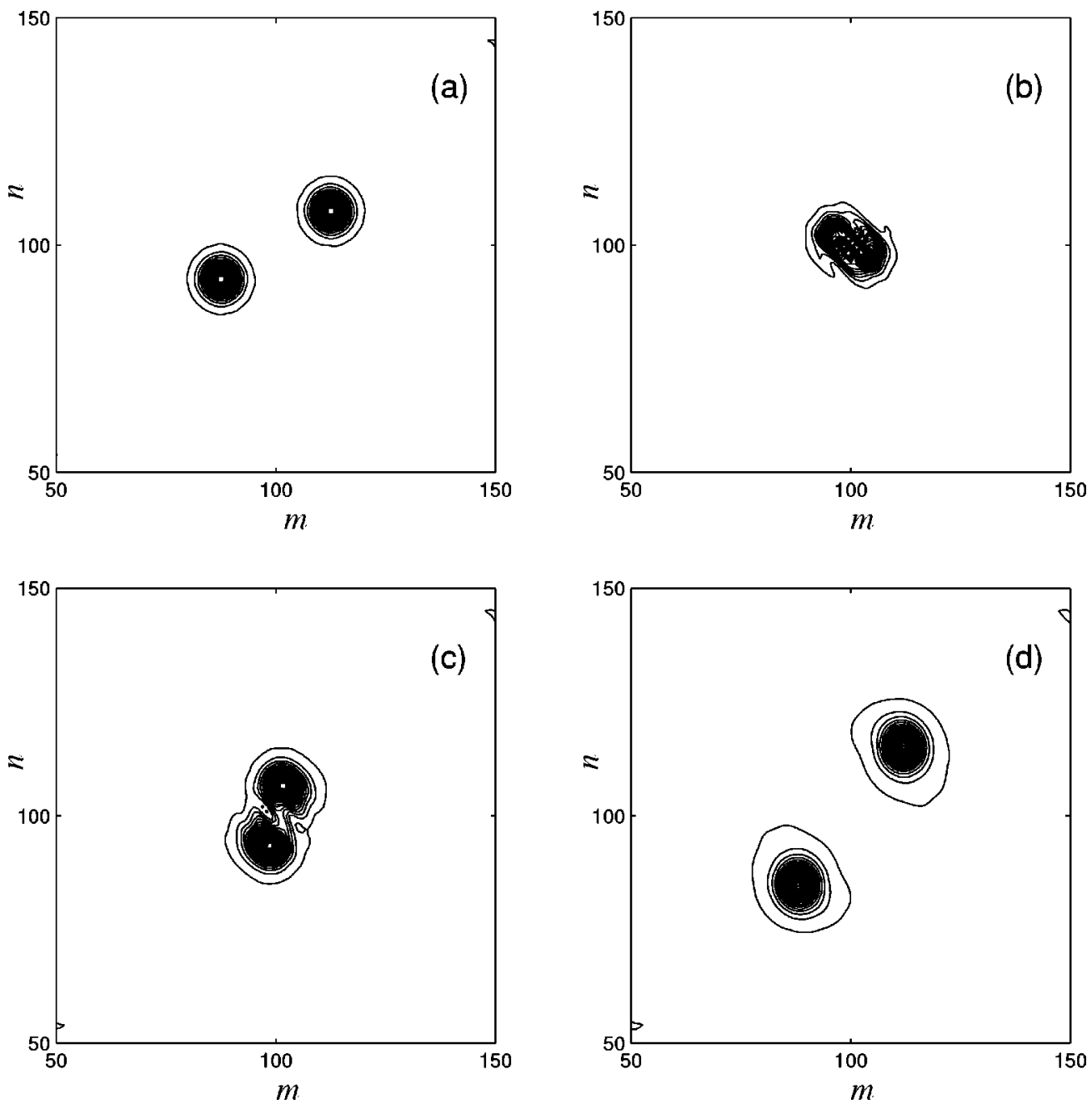

FIG. 15. Interaction of two extended polarons in the lattice with $\alpha=1, \beta=14$, and $\sigma=0.2$ shown by profile contours for the $u_{m n}$ polaron component at the time instants: (a) $\tau=200$, (b) $\tau=250$, (c) $\tau=275$, and (d) $\tau=325$. The direction of polaron propagation and their velocities are given by the vectors $\mathbf{k}=(0.5,0.5)$ and $\mathbf{s}=(0.192,0.192)$.

laronic state. Thus, the simple analytical arguments ${ }^{32}$ in the harmonic approximation for 1D standing polarons show that the binding energy per one electron is four times larger in the bipolaronic state than in the polaronic state with one electron (it is much easier for two electrons to dig a potential well when they are together than separated by a long distance). However, in the case of moving polarons, such a bound state will not be formed if the relative velocity of two polarons is too high, when the kinetic energy of the polarons exceeds the energy of their binding.

We collide the two moving polarons, the profiles of which are obtained by the minimization techniques described above. In order to find their initial profiles correctly, we notice that the probability of each electron localized separately on the lattice is $1 / 2$ instead of 1 . Therefore the normalization condition (19) in the minimization procedure should be changed accordingly, i.e., 1 should be replaced by $1 / 2$ for both the electrons which are sufficiently separated. We simulated the collision on the $200 \times 200$ square lattice and found that the polarons interact practically elastically for all initial velocities, except for very small ones when the duration of their interaction is very large. In the latter case, the collision was observed to be destructive. This can be explained as follows. At low velocities the time of the polaron interaction is quite long, resulting in strong effective perturbation of each polaron. Since the minimum of the variational energy (31) for extended polarons is very shallow (see Fig. 2), it is quite easy to "kick out" a polaron from this ground state. It may be possible that during long time the polaron will come back to this ground state, but it is difficult to observe this in numerical experiments due to long integration time and large lattice size. However, if we choose the parameter values that correspond to narrower polarons, their collision with these small velocities was observed to be nearly elastic. The results of the two-polaron collision with higher velocities are presented in Fig. 15. The polarons were started to move towards each other not along the same line, but parallelly with a sufficiently small distance between the lines of their motion. This distance was less than the width of the polaron, see Fig. 15. As illustrated by this figure, the interaction of the polarons is close to being elastic. Note that the time intervals at which the collision contours are shown in this figure are not equidistant. Finally, we would like to mention that the detailed interaction is really very complex and depends on several parameters. This is the subject for future studies and it is beyond the scope of the present paper. 


\section{CONCLUSIONS}

In this paper we have studied the polaron problem, i.e., the interaction of an extra electron (generally, a quantum quasiparticle) with the two-dimensional (2D) square lattice within the well-known Holstein model with local electronlattice coupling. This is the simplest model in the polaron theory and its solution is known when the on-site potential is harmonic. The results known for one dimension drastically differ from those in two or three dimensions. Thus, while for the 1D model the localized (polaron) solutions exist for any values of system parameters and there is the continuous transition from the small-polaron regime to the large one, in two or three dimensions the polaron solutions are known to exist only for sufficiently strong electron-lattice coupling. Moreover, these self-trapped states appear to be quite narrow, localized mainly at one lattice site. If the electron-lattice interaction is not strong enough, only completely delocalized solutions are possible. On the other hand, one could expect that if the displacement of the lattice particles from equilibria is somehow restricted, say, by a core, then a $2 \mathrm{D}$ polaron profile would become more extended. Therefore our goal was to consider the realistic situation when the on-site oscillators are anharmonic, containing a restricting core. In order to treat the model with such a potential, we have introduced a "logarithmic', approximation which, on one hand, still has the core as realistic potentials and, on the other hand, allows us to apply variational approximations for analytical investigations of the problem. As a result, we have shown that, in dependence on the system parameters, the self-trapped states with extended profiles can also exist. Such extended polarons have been shown to propagate uniformly on the 2D lattice. We have developed the numerical procedure to calculate moving polaron profiles. We have used these as initial data for simulations of the equations of motion showing that stable moving polarons of a certain extent are indeed possible. We have also obtained the diagram on the plane of system parameters which shows the areas of existence and nonexistence of the $2 \mathrm{D}$ polaron solutions.

We have also found that the presence of an anharmonicity "splits" the electron-lattice coupling parameter $g$ $=\chi^{2} / J M \omega_{0}^{2}$ [see Eqs. (8) and (29)], commonly used in the polaron theory, into the two parameters $\alpha$ and $\beta$ describing the two effects. The former parameter describes the depth of a potential well (compared to its width) that traps an external electron while the latter one determines the source strength for the lattice distortion created by the electron. In the harmonic limit both the equations of motion (9) and (10) are linear and, as result, the self-trapping constant $g$ is obtained just by the multiplication of these constants [see Eq. (29)]. Note that the nonlinearity of the polaron problem comes from this product. However, in the general case, these constants are separated and such a splitting should be generic in any "'anharmonic", polaron theory. Note also that the anharmonicity has its own parameter which differs from $\alpha$ and $\beta$. Finally, it should be mentioned that the results on the polaron mobility obtained in this paper are relevant to problems of electron transport in condensed-matter systems.

\section{ACKNOWLEDGMENTS}

Y.Z. wishes to acknowledge the financial support from the Danish Research Academy, and would like to express his thanks for the hospitality of the MIDIT Center and the Department of Mathematical Modelling of the Technical University of Denmark. Part of this work was supported by the Danish Natural Sciences Foundation (SNF). Stimulating and useful discussions with Yu. B. Gaididei, A. V. Savin, and J. Schjødt-Eriksen as well as their suggestions about the process of this investigation, are gratefully acknowledged.
${ }^{1}$ L. Landau, Phys. Z. Sowjetunion 3, 664 (1933).

${ }^{2}$ S. I. Pekar, J. Phys. (Moscow) 10, 341 (1946); Untersuchungen über die Electronentheorie der Kristalle (Akademie-Verlag, Berlin, 1954).

${ }^{3}$ H. Fröhlich, Proc. R. Soc. London, Ser. A 215, 291 (1952).

${ }^{4}$ T. Holstein, Ann. Phys. (N.Y.) 8, 325, 343 (1959).

${ }^{5}$ Y. Toyozawa, Prog. Theor. Phys. 26, 29 (1961).

${ }^{6}$ E. I. Rashba, Zh. Eksp. Teor. Fiz. 54, 542 (1968) [Sov. Phys. JETP 27, 292 (1968)].

${ }^{7}$ D. Emin and T. Holstein, Phys. Rev. Lett. 36, 323 (1976).

${ }^{8}$ D. Emin, Phys. Today 35, (6), 34 (1982).

${ }^{9}$ A. S. Davydov and N. I. Kislukha, Phys. Status Solidi B 59, 465 (1973).

${ }^{10}$ A. C. Scott, Phys. Rev. A 26, 578 (1982).

${ }^{11}$ A. S. Davydov, Solitons in Molecular Systems (Reidel, Dordrecht, 1985).

${ }^{12}$ For a review and other references, see e.g., B. Gerlach and $\mathrm{H}$. Löwen, Rev. Mod. Phys. 63, 63 (1991); A. C. Scott, Phys. Rep. 217, 1 (1992).

${ }^{13}$ J. C. Eilbeck, P. S. Lomdahl, and A. C. Scott, Phys. Rev. B 30, 4703 (1984); Physica D 16, 318 (1985).

${ }^{14}$ Y. Zhao, D. W. Brown, and K. Lindenberg, J. Chem. Phys. 100, 2335 (1994); 106, 2728 (1997).
${ }^{15}$ A. La Magna, R. Pucci, G. Piccitto, and F. Siringo, Phys. Rev. B 52, 15273 (1995).

${ }^{16}$ P. S. Lomdahl and D. J. Srolovitz, Phys. Rev. Lett. 57, 2702 (1986).

${ }^{17}$ J. Pouget, S. Aubry, A. R. Bishop, and P. S. Lomdahl, Phys. Rev. B 39, 9500 (1989).

${ }^{18}$ J. Pouget, Phys. Rev. B 43, 3575 (1991); 43, 3582 (1991); 46, 10554 (1992).

${ }^{19}$ J. M. Tamga, M. Remoissenet, and J. Pouget, Phys. Rev. Lett. 75, 357 (1995)

${ }^{20}$ G. Kalosakas, S. Aubry, and G. P. Tsironis, Phys. Rev. B 58, 3094 (1998).

${ }^{21}$ A. M. Kosevich, Nonlinear Mechanics of Crystals (Institute for Physics of Metals, Sverdlovsk, 1975) (in Russian).

${ }^{22}$ J. W. Grantham, H. M. Gibbs, G. Khitrova, J. F. Valley, and Xu Jianjin, Phys. Rev. Lett. 66, 1422 (1991).

${ }^{23}$ B. Luther-Davies, R. Powles, and V. Tikhonenko, Opt. Lett. 19, 1816 (1994).

${ }^{24}$ V. Tikhonenko, J. Christou, and B. Luther-Davies, J. Opt. Soc. Am. B 12, 2046 (1995); Phys. Rev. Lett. 76, 2698 (1996).

${ }^{25}$ F. Vidal and T. W. Johnston, Phys. Rev. E 55, 3571 (1997).

${ }^{26}$ L. Bergé, M. R. Schmidt, J. Juul Rasmussen, P. L. Christiansen, 
and K. Ф. Rasmussen, J. Opt. Soc. Am. B 14, 2550 (1997).

${ }^{27}$ J. Schjødt-Eriksen, M. R. Schmidt, J. Juul Rasmussen, P. L. Christiansen, Yu. B. Gaididei, and L. Bergé, Phys. Lett. A 246, 423 (1998).

${ }^{28}$ J. Schjødt-Eriksen, Master's thesis, DTU, Lyngby, 1998.

${ }^{29}$ E. W. Laedke, K. H. Spatschek, V. K. Mezentsev, S. L. Musher, I. V. Ryzhenkova, and S. K. Turitsyn, JETP Lett. 62, 677 (1995). Since in our case the wave function $\psi_{m n}$ is normalized to unity [see Eq. (2)], instead of the integral of motion $\Sigma_{m, n}\left|\psi_{n m}\right|^{2}$, we formulate the stability criterion in terms of the parameters $\alpha$ and $\beta$.

${ }^{30}$ P. L. Christiansen, Yu. B. Gaididei, K. Ф. Rasmussen, V. K. Mezentsev, and J. Juul Rasmussen, Phys. Rev. B 54, 900 (1996).

${ }^{31}$ P. L. Christiansen, A. V. Savin, and A. V. Zolotaryuk, J. Comput. Phys. 134, 108 (1997).

${ }^{32}$ See, e.g., A. V. Zolotaryuk, A. Mistriotis, and E. N. Economou, Phys. Rev. B 48, 13518 (1993). 